NISTIR 8305

\title{
Survey of Drone Usage in Public Safety Agencies
}

\author{
Hien V. Nguyen \\ Terese W. Manley \\ Kamel S. Saidi
}

This publication is available free of charge from:

https://doi.org/10.6028/NIST.IR.8305

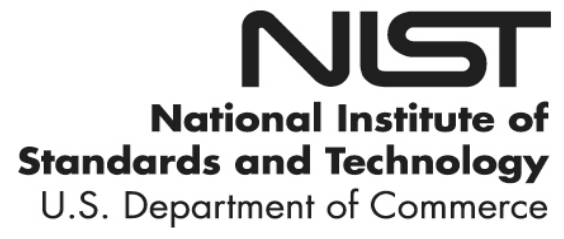




\title{
Survey of Drone Usage in Public Safety Agencies
}

\author{
Hien V. Nguyen \\ Terese W. Manley \\ Public Safety Communications Research Division \\ Communications Technology Laboratory \\ Kamel S. Saidi \\ Intelligent Systems Division \\ Engineering Laboratory
}

This publication is available free of charge from:

https://doi.org/10.6028/NIST.IR.8305

April 2020

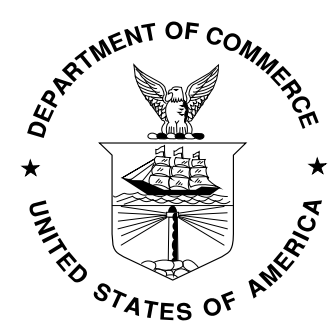

U.S. Department of Commerce Wilbur L. Ross, Jr., Secretary

National Institute of Standards and Technology Walter Copan, NIST Director and Undersecretary of Commerce for Standards and Technology 
Certain commercial entities, equipment, or materials may be identified in this document in order to describe an experimental procedure or concept adequately. Such identification is not intended to imply recommendation or endorsement by the National Institute of Standards and Technology, nor is it intended to imply that the entities, materials, or equipment are necessarily the best available for the purpose.

The National Institute of Standards and Technology Research Protections Office reviewed the protocol for this project and determined it meets the criteria for "exempt human subjects research" as defined in 15 CFR 27, the Common Rule for the Protection of Human Subjects.

National Institute of Standards and Technology Interagency or Internal Report 8305 Natl. Inst. Stand. Technol. Interag. Intern. Rep. 8305, 26 pages (April 2020)

This publication is available free of charge from: https://doi.org/10.6028/NIST.IR.8305 


\begin{abstract}
The Public Safety Communications Research (PSCR) Division at the National Institute of Standards and Technology (NIST) conducted a survey to explore ways in which drones, or Unmanned Aircraft Systems (UAS), are currently being used in public safety operations and identify potential opportunities for drones to improve operations in the future. The participants of the survey included 183 first responders, and the responses were collected in the time period of April through July 2019. The results and further analysis of the survey are explored in this report.
\end{abstract}

\title{
Keywords
}

Broadband; drones; first responder; public safety; public safety communications; unmanned aircraft system (UAS); wireless communications.

\section{Measurement Units}

The U.S. customary units (a.k.a. imperial units) are used throughout this document. The International System of Units (a.k.a. SI Units) was not used in the UAS survey or in this document because the intended audience, the public safety community, does not currently use SI Units in UAS terminology. 


\section{Table of Contents}

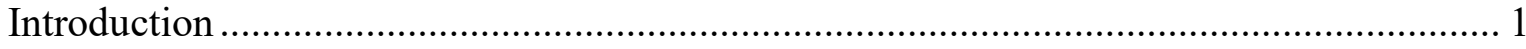

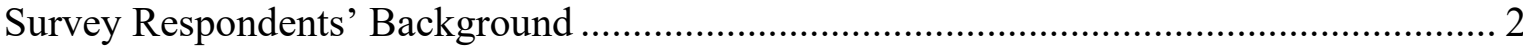

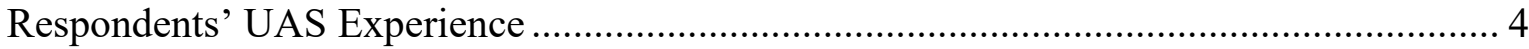

The Need for Wireless Communications ..................................................................... 7

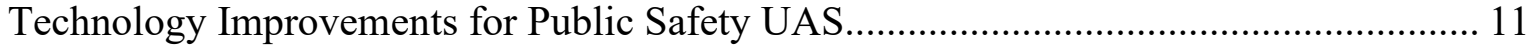

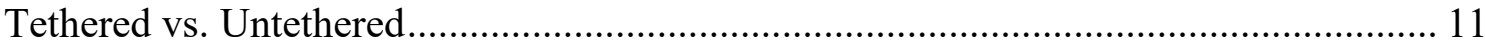

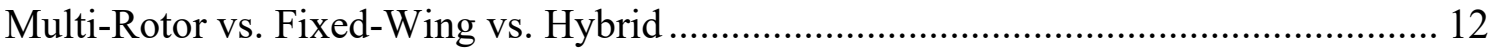

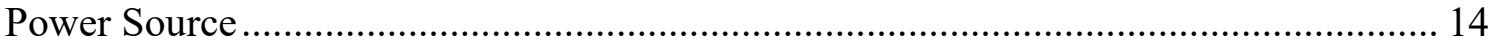

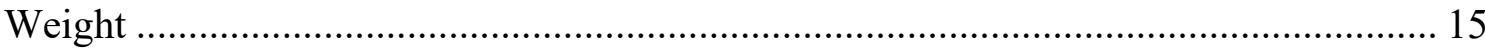

Other UAS Features Needed for Public Safety ............................................................... 16

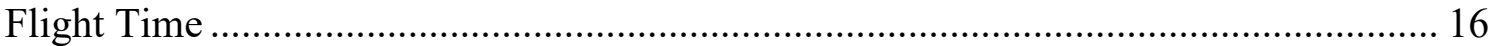

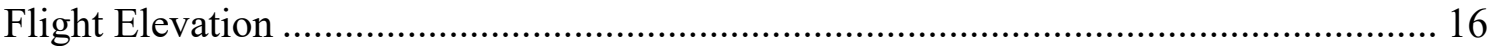

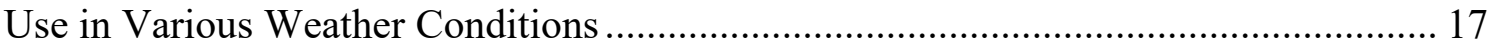

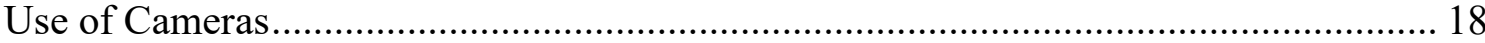

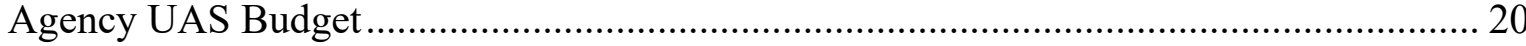

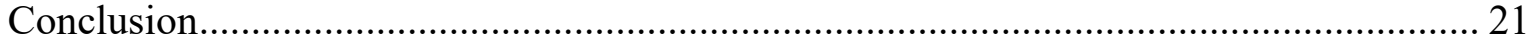

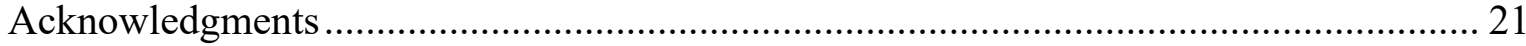




\section{Introduction}

The National Institute of Standards and Technology (NIST) is the national physical laboratory for the United States. NIST works with the private sector, other government agencies, and universities to develop and apply the technology, measurements and standards needed for new and improved products and services. The Public Safety Communications Research (PSCR) Division is a division under NIST and, as a technology advisor to public safety organizations, plays an important role in providing technical expertise and evaluating forwardthinking technologies that first responders need for their wireless communications. One of the specific areas of research that PSCR is addressing with this survey, Resilient Systems, is examining ways to ensure first responders can operate more effectively in areas without macro cellular network coverage, by bringing the coverage with them. Since disasters may limit available power sources, backhaul, or cellular towers in a damaged area, PSCR is exploring ways to fill that gap by giving first responders control of their communications systems through the deployment of Unmanned Aircraft Systems (UAS), or drones, carrying wireless networks.

PSCR regularly meets with members of the first responder community to ask their advice about matters pertaining to wireless communications and to help inform the direction of their research. PSCR conducted a survey in 2019 to ask America's first responders how they use drones in their current or past operations and to identify potential benefits for using drones in the future to support wireless communications during an incident. Over the past decade, public safety teams have begun to deploy drones on missions in hard-to-reach areas or otherwise challenging conditions. PSCR is evaluating deployable systems to support these missions and actively looking at drones as a new way for first responders to support wireless communications for public safety personnel. Specifically, PSCR is conducting research where drones would carry a network communications system for extraordinary incidents. Deployable systems research performed by NIST PSCR, guided by results from this survey, will continue to shape UAS research efforts and the mission of advancing public safety wireless communications.

The recipients of this survey included first responders and public safety drone experts with varied levels of UAS experience. Survey questions were designed to collect data based on their first-hand experience with drones as it relates to incident operations and the value of drones as an additional tool for first responders. The survey was sent to a distribution list of almost 900 public safety-related professionals, garnering responses from 183 people (a 20\% response rate) from April through July 2019. The survey was designed to be anonymous and did not collect email addresses or names. Although unlikely, there is a possibility that respondents took the survey more than once. Also, some respondents may be federal, state or local government employees working on public safety-related projects. This survey was conducted by NIST PSCR under OMB Control \#0693-0033. 


\section{Survey Respondents' Background}

UAS technology is slowly being incorporated into public safety operations and deployed for uses including wireless communications. For this survey, PSCR targeted first responders nationwide who are known to have experience or expertise in UAS operations, or likely to have been exposed to UAS technology.

The survey identified individuals with job titles representing all levels of their organizations, with $86 \%$ having over five years of experience in those jobs. Specifically, some of the job positions/titles identified were:

- Lieutenant

- Battalion Chief

- Emergency Management Coordinator

- Fire Chief

- Engineer
- Captain

- Assistant Chief

- Communications Manager

- Chief Pilot

- Fire Marshal

- SWAT Officer

Note: $97.8 \%$ of those surveyed answered the question: "What is your position/title within your organization?"

PSCR collected data from respondents in public safety organizations representing most jurisdictions (city, county, state, regional, federal), with the majority of respondents belonging to the following disciplines:

- Law Enforcement (54\%)

- Fire Fighting (44\%)

- $\quad$ EMS (Emergency Management Service) (26\%)

- Rescue Services (24\%)

- Emergency Management (11\%)

Note: $96.7 \%$ of those surveyed answered the question: "What type of public safety organization are you currently with?" Respondents could select multiple answers for this question.

The survey also identified the respondents' geographic operational environments, which is important for understanding the types of air space required:

- Rural (68\%)

- Suburban $(68 \%)$

- Urban (61\%)

- Wildland $(43 \%)$

- Mountains (25\%)

- Desert (13\%)

Note: $97.8 \%$ of those surveyed answered the question: "What type of geographic environments does your department typically operate in?" Respondents could select multiple answers for this question. 
As a final survey question about the respondents' backgrounds, PSCR sought to understand how many years of experience the respondents had in their current public safety jobs. The results showed that nearly $74 \%$ of respondents had more than 10 years of experience in their current jobs.

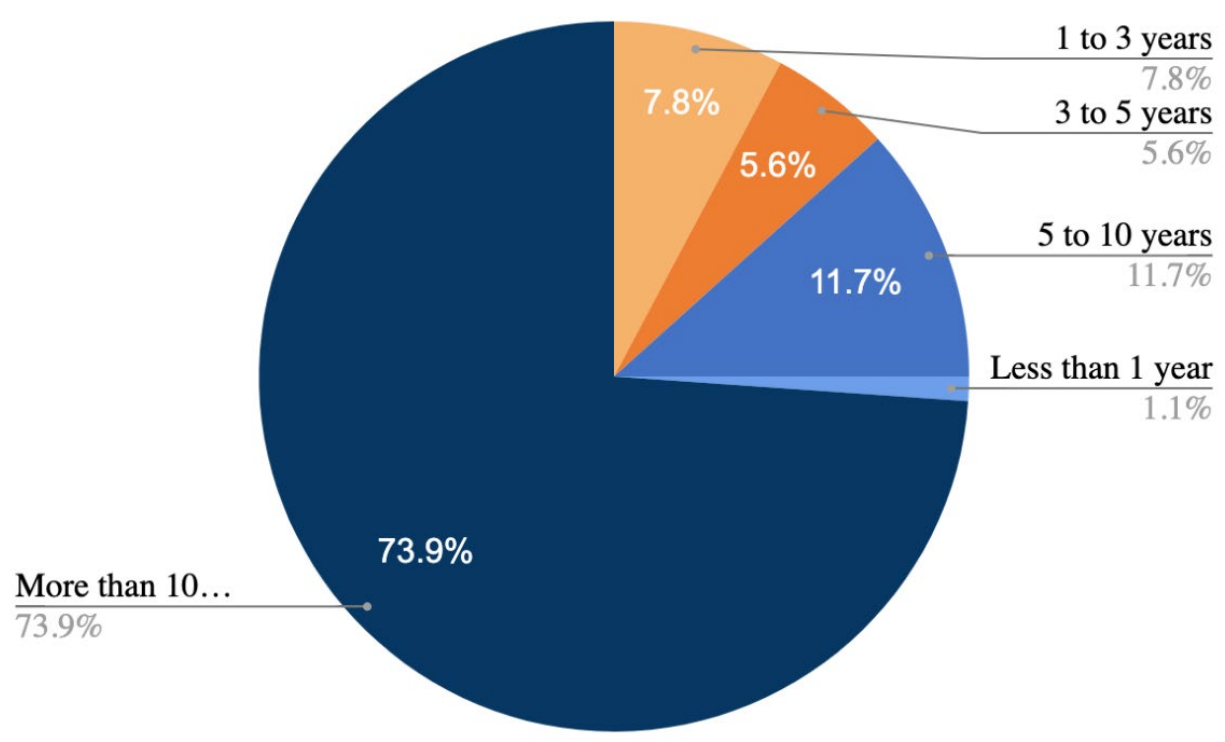

Figure 1. Displays answers to the question: "How many years of experience do you have in your current line of work?" Of those surveyed, $98.4 \%$ answered this question.

Since the depth of public safety experience of the respondents was important for PSCR in qualifying the answers in the survey, a follow-up question asked if they had work experience in public safety previous to their current lines of work. Of the respondents who answered "Yes" to having previous public safety experience, $71.2 \%$ stated their previous experience spanned more than five years. 


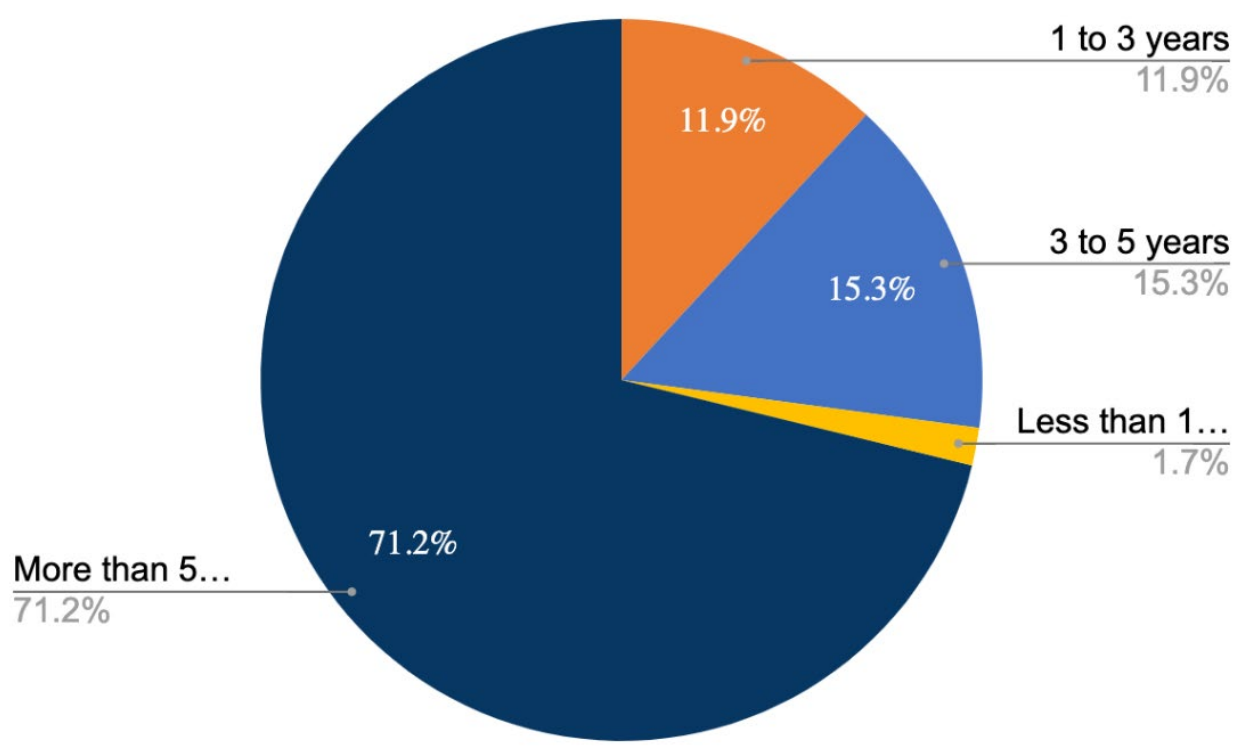

Figure 2. Displays answers to the question: "How many years of experience did you have with your prior public safety organization(s)?" Of those surveyed, $64.5 \%$ answered this question.

\section{Respondents' UAS Experience}

The previous set of questions were designed to gain a better understanding of the respondents' backgrounds, organizations, and job skills for the people represented in the survey. The next set of questions focused on the respondents' UAS experience.

When asked whether they had any experience flying drones, nearly all of the 183 respondents answered with $72.5 \%$ answering "Yes." Respondents had experience ranging from no drone experience to advanced skills, such as building and designing drones. Of those with experience, only $12.9 \%$ had more than five years of experience flying drones. Below is a subset of comments offered by respondents, in addition to their number of years of experience, as shown in Figure 3.

- Minimal experience or less than one year of experience

- Awaiting training as a drone pilot

- Starting a drone operation in their organization

- Manages or oversees a team of drone operators

- Instructor pilot or military trained

- Built drone aircraft

- Hobbyist with experience flying off-the-shelf drones

- FAA (Federal Aviation Administration) Part 107 Certified or receiving certification 


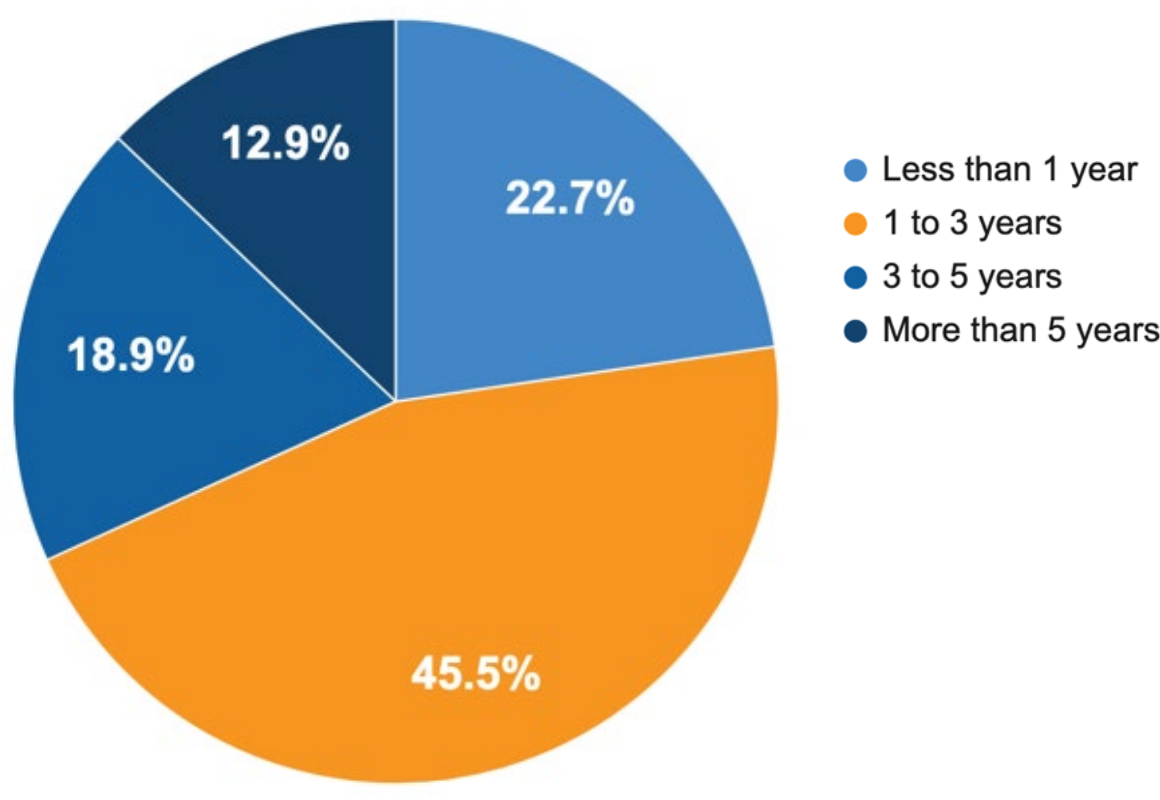

Figure 3. Displays answers to the question: "How many years of drone flying experience do you have?" Of those surveyed, $72.1 \%$ answered this question.

Then, PSCR took a deeper dive into public safety UAS programs. For the respondents and their departments, the next two questions identified the status of their UAS programs. The survey found that over $62 \%$ of respondents stated that their departments had a UAS program, $97 \%$ stated that their organizations had a UAS pilot, and $78 \%$ stated that their UAS programs were established within the past three years (as of June 2019). The answers to these questions are reflected in Figure 4 and Figure 5 below, respectively.

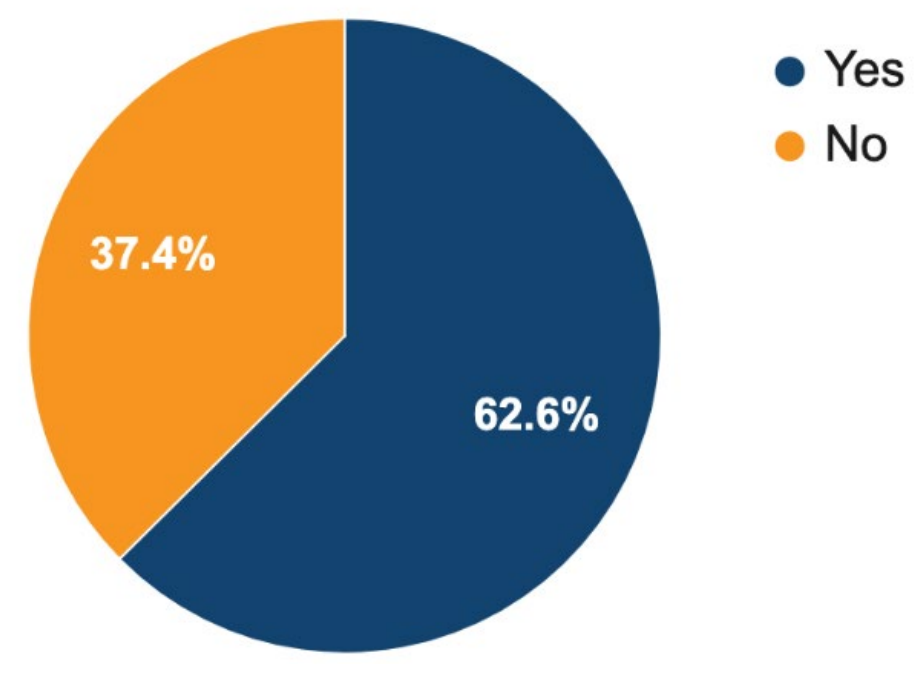

Figure 4. Displays answers to the question: "Have you worked (or do you currently work) in a public safety department that has a drones program?" Of those surveyed, $99.5 \%$ answered this question. 


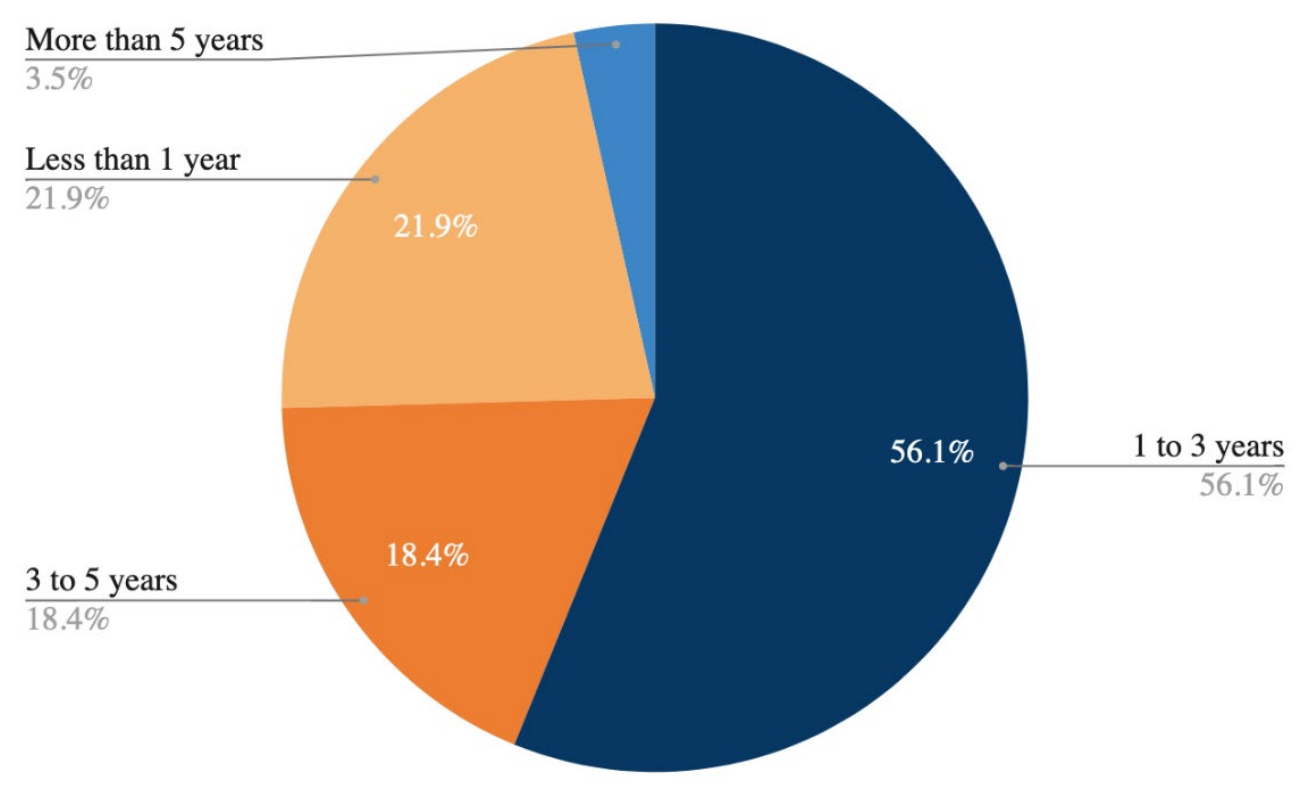

Figure 5. Displays answers to the question: "How long has your department had (or did your department have) a drones program?" Of those surveyed, $62.3 \%$ answered this question. 


\section{The Need for Wireless Communications}

The next set of questions were designed to identify the need for cellular broadband communications in areas where first responders operate. PSCR focused on missions where cellular broadband was not available and asked respondents whether there was a need for cellular communications. When asked if respondents were involved in missions without cellular broadband available, over $69 \%$ answered "Yes." Of those respondents, over $94 \%$ responded they would have wanted cellular broadband available during those missions.

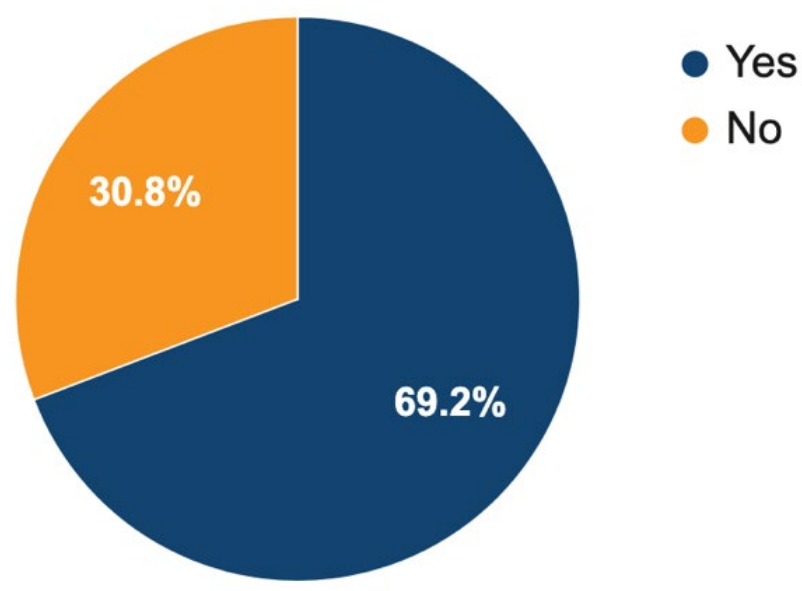

Figure 6. Displays answers to the question: "Have you been involved in any missions during which cellular broadband communications (voice and/or data) were not available (e.g., the wireless infrastructure was down or you were in a remote area where wireless infrastructure didn't exist)?" Of those surveyed, $99.5 \%$ answered this question.

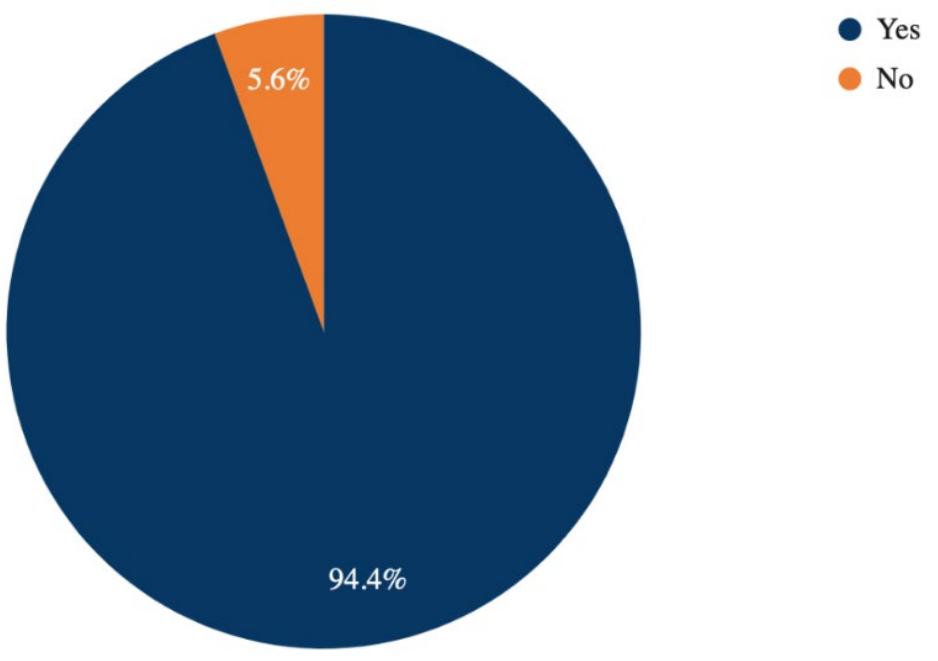

Figure 7. Displays answers to the question: "Where cellular broadband communications were not available, was there a need for, or would you have wanted, wireless communications?" Of those surveyed, $69.8 \%$ answered this question. 
Survey respondents were then asked to describe an incident or mission where wireless communications were not available, in order to build upon this scenario in later questions. Respondents were asked to describe further the environment and the reason a communications system was not available during the mission. A representative selection of answers is shown below.

- Search and rescue missions during natural disasters, hurricanes, wildfires, floods, warrant services, crime scenes, and wildland fires. These search and rescue missions are carried out in various environments, including rural, very remote, rugged terrain, and mountainous conditions.

- Crime scene documentation

- Earthquake

- 911 terrorist attack on the Pentagon

- Patrol in urban areas with residential dwellings, hospitals, parking garages, and highrise buildings

- Firefighting and fire scene reconnaissance/search in commercial and rural areas

- Flooding and flood response

- Hurricane response and recovery

- Missing persons and manhunts carried out in various environments, ranging from residences to national forests, rural, very remote, and mountainous situations

- Border enforcement and locating illegal aliens in rural areas

- Drug raid

- Vehicle collision reconstruction

- National disasters

- Tornado recovery

- Train derailment

- Wildland fire

- Hazmat situation

- Plane crashes in rural and mountainous areas

- Post-disaster assessment

Note: $65.6 \%$ of those surveyed answered the question: "Briefly list the kind of mission (such as for a missing person), the type of environment (such as dwelling, high-rise buildings, fields, rugged mountainous terrain), and the reason communications systems were not available."

In order to understand better how a UAS could be used to improve the previously described missions, respondents were asked about the added benefit to their missions if they had access to cellular broadband communications. A representative selection of answers is shown below.

- Ability to download maps in real time, update mobile apps, and use third-party software to stream feedback to the station

- Access to current weather forecast, location services, data, online maps, information, subject matter experts, and evidence transmission 
- Better control, better view of the situation, and resource allocation

- Broadband data

- Communication and coordination with command, hospitals, other agencies, and the emergency command center

- Damage assessment

- Faster communications and transfer of data

- Flight reconnaissance

- Incident communications and mission planning

- Increased situational awareness

- Live streaming of video and images to command and responders

- Recovery of lost bodies

- Resource tracking

- Video back-haul

- Uploading images and video to the cloud

- Increased safety and efficiency

- Enhance operations

- Free up radio channels

- Ability to have private conversations

- Logistical support for other agencies

- Faster mutual aid response

- GPS monitoring

Note: $59.6 \%$ of those surveyed answered the question: "Please describe what the added benefit to the mission would have been."

To learn how UAS technology might improve public safety's needs, survey respondents were asked whether having continuous cellular communications was a necessity in the types of missions where they were involved. Of the responses, $77 \%$ answered "Yes" and 22\% answered "No."

Below is a representative selection of explanations from those respondents who answered "Yes."

- Increase safety of the crew, reduce property loss and accomplish the mission.

- Improve situational awareness and capabilities exponentially.

- Coordination and communication are critical in emergency operations.

- Continuous cellular communication is considered "mission critical"; without cellular we rely on satellite for data.

- Drone video distribution is dependent on cellular coverage.

- Updated mapping and real-time video streaming.

- Good cellular data coverage is required to coordinate with other agencies who don't carry radios.

- Ongoing need for information exchange between line personnel, incident command, dispatch and outside experts.

- Good to connect out-of-area resources who are responding. 
- Current operation requires deployment of VSAT (Very Small Aperture Terminal) and BGAN (Broadband Global Area Network) for connectivity in areas with no coverage.

- Public safety dispatch is more efficient by utilizing mobile data terminals tied to a CAD (Computer-Aided Design) system.

- Alternative communication systems can be expensive and difficult to maintain.

- Daily operations and critical response have become more data-driven and reliant upon cellular communications.

- Without cellular communications, there can be safety issues, time delays, and a lack of mobile data when needed the most.

Below is a representative selection of explanations from those respondents who answered "No."

- Basic communications are provided by Public Safety P25 LMR (Land Mobile Radio), which provides more reliable coverage in mountainous areas.

- The job is getting done without it in rural areas, although it may become necessary in the future due to changing operations that will leverage broadband.

- It offers enhancements, but the mission can continue without it, albeit a little slower.

- Backup plans are in place for data processing and messaging without data comms.

- Increased risk to equipment and personnel safety if equipment software is not updated.

- Drones don't need cell coverage for a rescue mission, but it could evolve.

- Data is not mission critical for most missions or for basic operations.

Note: Of the $99.5 \%$ of respondents who answered yes or no, $68.3 \%$ provided an explanation when answering this question: "Would you consider continuous cellular communications a necessity in the types of missions that you are involved in? If Yes, please explain your answer." 


\section{Technology Improvements for Public Safety UAS}

Since PSCR is focused on advancing wireless communications for public safety, it was important to understand how large the coverage area would need to be in order to support a typical incident. In other words, what is the wireless coverage area necessary to support first responders in an environment that lacks a sufficient wireless infrastructure? Addressing this question, respondents estimated the cellular coverage area that might be needed to support the previously described mission. The answers varied amongst agencies, with almost $52 \%$ suggesting they would need coverage between 1 and 5 miles. The complete survey results are shown below in Figure 8.

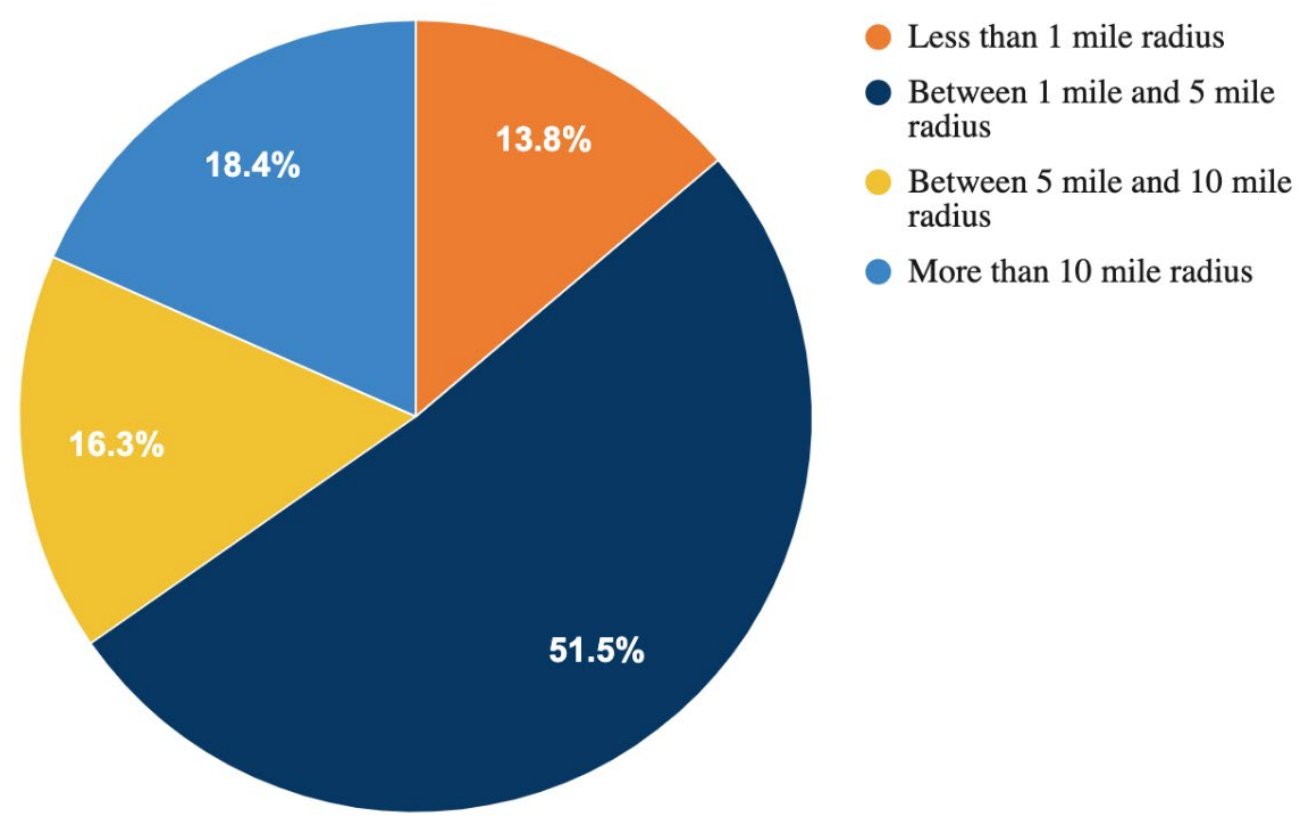

Figure 8. Displays answers to the question: "What size cellular broadband coverage area would you need surrounding a single mission area in order to support that mission?" Respondents could select multiple answers for this question, and $98.4 \%$ of those surveyed answered this question.

\section{Tethered vs. Untethered}

With known operational advantages and disadvantages to both tethered and untethered drones, PSCR sought to understand whether a drone would be more useful for their mission if it were tethered to a ground control station with a cable or untethered. Survey respondents were also asked to explain their preferences for using tethered, untethered, or mix of both types of drones in their operations. The survey results demonstrated that $61 \%$ of organizations prefer a mix of both configurations. 


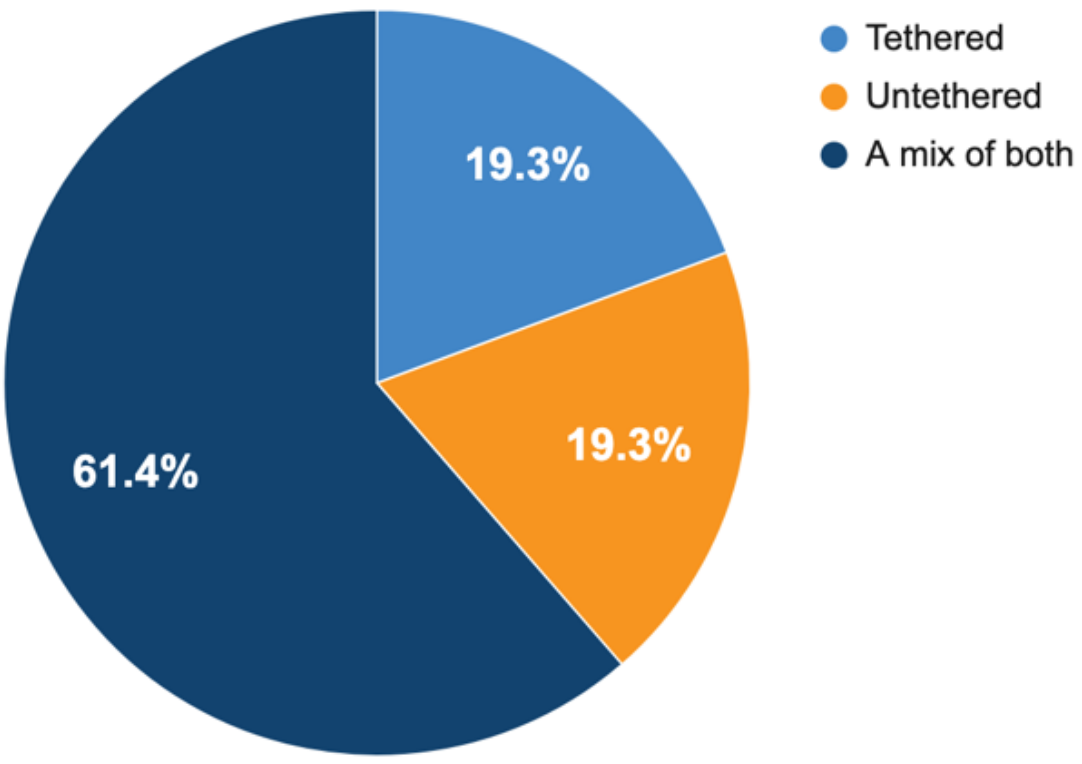

Figure 9. Displays answers to the question: "If a drone (that can provide continuous cellular broadband coverage) could be either tethered to a ground control station with a cable (supplying continuous power to the drone and communications between the ground station and the drone), or untethered (i.e., completely wireless), which one would be more useful for your organization or missions?" Of those surveyed, $62.3 \%$ answered this question.

PSCR received the following responses to help explain the reasoning behind respondents' choices of tethered vs. untethered for their missions.

- More flexibility with untethered

- Tethered provides additional flying time

- Tethered provides long-term communications support

- Untethered for area-wide observation

- Power is always an issue

- Concerned about flying a drone in areas with trees

- Prefer tethered because another operator and observer is not needed

- Untethered is a much more versatile platform

- Length of flying time and payload capacity

Note: $50.8 \%$ of those surveyed answered the question: "Please explain your choice for tethered, untethered, or both."

\section{Multi-Rotor vs. Fixed-Wing vs. Hybrid}

Next, PSCR investigated which aircraft technology was best suited for public safety missions where continuous cellular broadband coverage might be needed. When survey respondents were asked if they had a preference for multi-rotor, fixed-wing, or hybrid UAS, survey results revealed that almost $75 \%$ of the respondents answered "Yes," indicating a preference for the type of drone design used for their missions. 


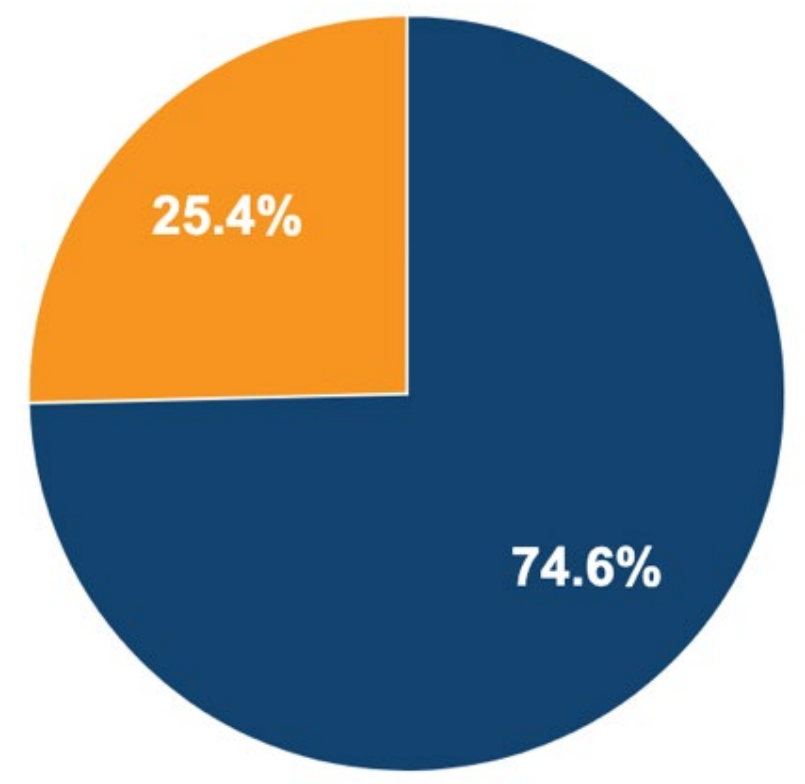

- Yes

- No

Figure 10. Displays answers to the question: "If a drone (that can provide continuous cellular broadband coverage) could be either an untethered multi-rotor drone that can take off and land vertically, an untethered fixed-wing drone that can take off and land horizontally, or an untethered hybrid drone that can take off and land vertically and fly horizontally, would you choose one type over another?" Of those surveyed, $62.3 \%$ answered this question.

The respondents expanded on their answers to the question by indicating their preference to one of the three types of UAS designs, giving the following answers:

- Either they had no preference or did not give a relevant answer (46\%)

- Preferred multi-rotor UAS $(29 \%)$

- Preferred fixed-wing UAS $(1 \%)$

- Preferred hybrid UAS (14\%)

- Vertical take-off and landing is crucial to their missions (regardless of whether the aircraft was a multi-rotor or a fixed-wing) $(11 \%)$

Note: $51.4 \%$ of those surveyed answered the question: "Please explain your answer and your drone preference."

When asked about the logistical and operational considerations for choosing one type of UAS design over another, the responses varied as shown in the following comments:

- Flight time

- Multiple missions and longer flight time

- Total manpower

- Cost and reliability

- Distance to cover and length of time aloft 
- Battery consumption

- Operating endurance

- Amount of space for launch and recovery

- Ease of use and implementation

- High quality and efficiency

- Flight pilot's skills

Note: $50.3 \%$ of those surveyed answered the question: "What are your logistical and operational considerations for choosing one type over another?"

\section{Power Source}

With recent technological advancements in batteries, liquid fuel and available power sources for drones, PSCR was interested in evaluating whether respondents would consider using alternative power sources (such as hydrogen fuel cells) in their drone operations, and $70 \%$ responded "Yes."

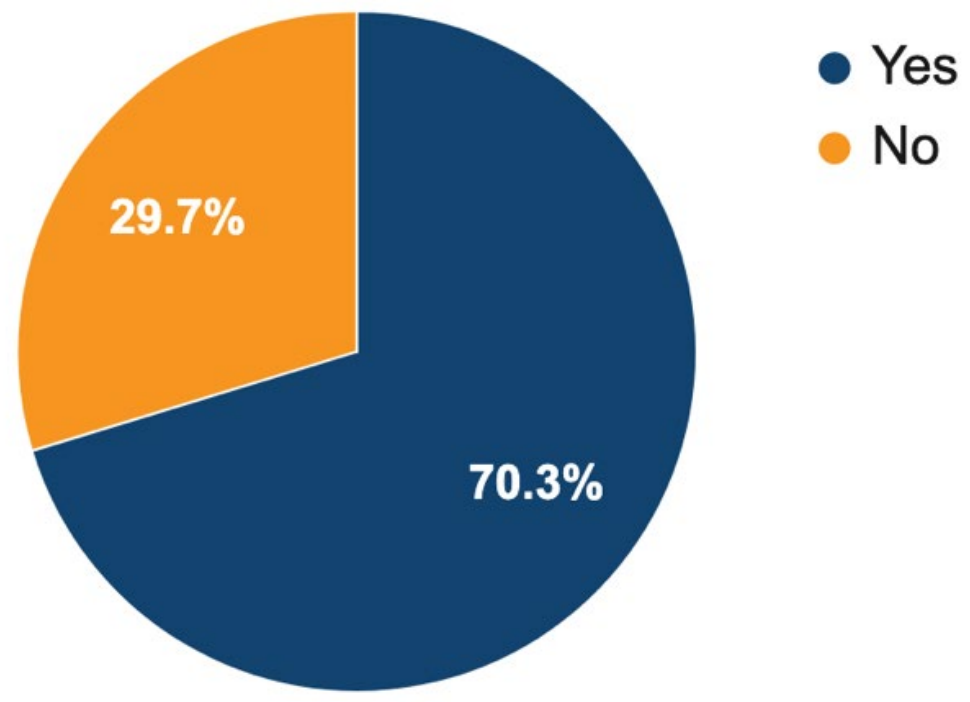

Figure 11. Displays answers to the question: "Would you consider other power sources such as hydrogen fuel cells, etc.?" Of those surveyed, $60.7 \%$ answered this question.

When asked about their preferred power source for drones when used to provide continuous cellular broadband coverage, respondents gave the following answers:

- Electric (50\%)

- Hybrid $(24 \%)$

- No preference or not relevant (17\%)

- Mission dependent (5\%)

- Liquid fuel (2\%)

- Whatever power source is most available $(2 \%)$ 
Note: $57.4 \%$ of those surveyed answered the question: "What would be your preferred power source for drones that can provide continuous cellular broadband coverage (for example, all electric, liquid fuel, or a hybrid electric/fuel)?"

\section{Weight}

Modern, small UAS technology offers a trade-off between flight time and payload capacity. Larger drones can often carry more energy, consequently, they can potentially stay in the air longer. However, drones that weigh 55 pounds or more require special waivers from the FAA in order to fly in the National Air Space. To understand whether first responders are reluctant to use larger, heavier drones for their missions, they were asked the following question: "If a drone that can provide continuous cellular broadband coverage were available and weighed more than $\mathbf{5 5}$ pounds, would you consider using it?" Slightly more than $68 \%$ of survey respondents answered "Yes" to considering this type of drone and, when asked to explain their answers, the responses varied (a sampling is shown below).

- More paperwork and a special permit is required.

- Time in the air (may provide the) benefit for drones over 55 pounds.

- Additional cost is an issue.

- Too big and heavy.

- If the drone was able to fill the capacity gap, then the extra weight and paperwork would not be an issue.

- (We would consider) whatever provides better coverage for an operation.

- The larger drone makes it difficult to coordinate (in FAA) airspace.

- Too big and heavy for fast deployment.

- (Yes but) more training and upkeep (is required).

Note: $61.7 \%$ of those surveyed answered the question: "If a drone that can provide continuous cellular broadband coverage were available and weighed more than $55 \mathrm{lbs}$. (which requires special waivers from the FAA to fly), would you consider using such a drone?" Of those surveyed, 49.2\% answered the question: "Why or why not?" 


\section{Other UAS Features Needed for Public Safety}

The survey continued with questions designed to examine other drone features that a first responder would need in the previously described mission, where a drone is deployed to provide continuous broadband coverage to an area.

\section{Flight Time}

When asked how long they would anticipate needing a drone to stay in flight during a mission, almost $100 \%$ of the 183 survey participants responded. The most popular answer showed that nearly $64 \%$ of respondents would need a drone to stay in the air continuously for more than 120 minutes. Other responses are indicated in Figure 12.

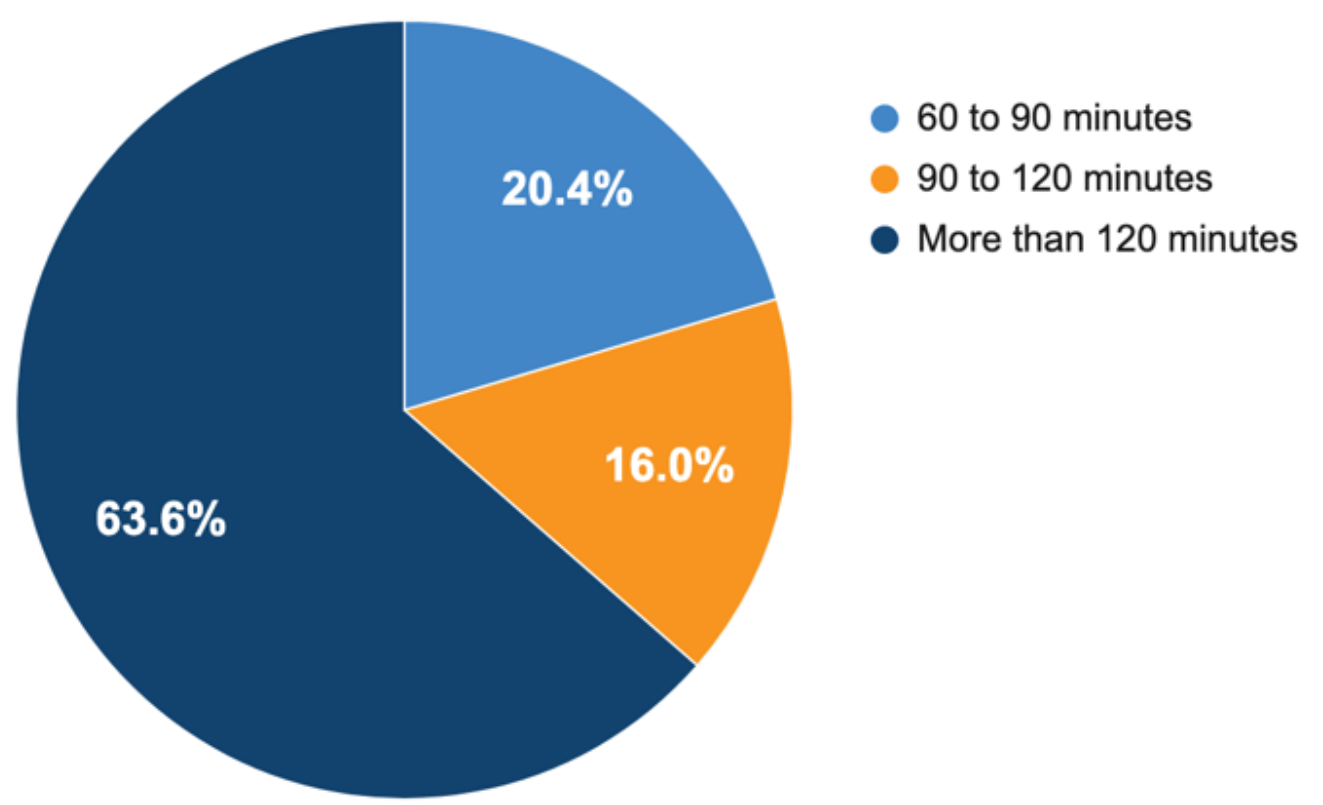

Figure 12. Displays answers to the question: "If drones could stay in the air indefinitely and a drone was able to provide continuous cellular broadband coverage for first responders in areas where coverage was not available, approximately how long would you anticipate needing the drone to stay in the air during such a mission?" Of those surveyed, $99.5 \%$ answered this question, and respondents could vote on multiple answers.

\section{Flight Elevation}

Since the maximum flight elevation above ground level (AGL) for small UAS under FAA requirements is 400 feet (under rules for Part 107 and with no waivers), it was important for PSCR to know if there was an interest amongst public safety organizations to fly below this height and avoid requirements for an FAA waiver. Survey results identified that there is an interest. Respondents were asked what they considered to be a safe minimum AGL for such a drone, and $90 \%$ of the 183 survey participants responded that the drone would be safe flying at an altitude of 400 feet AGL and below. 


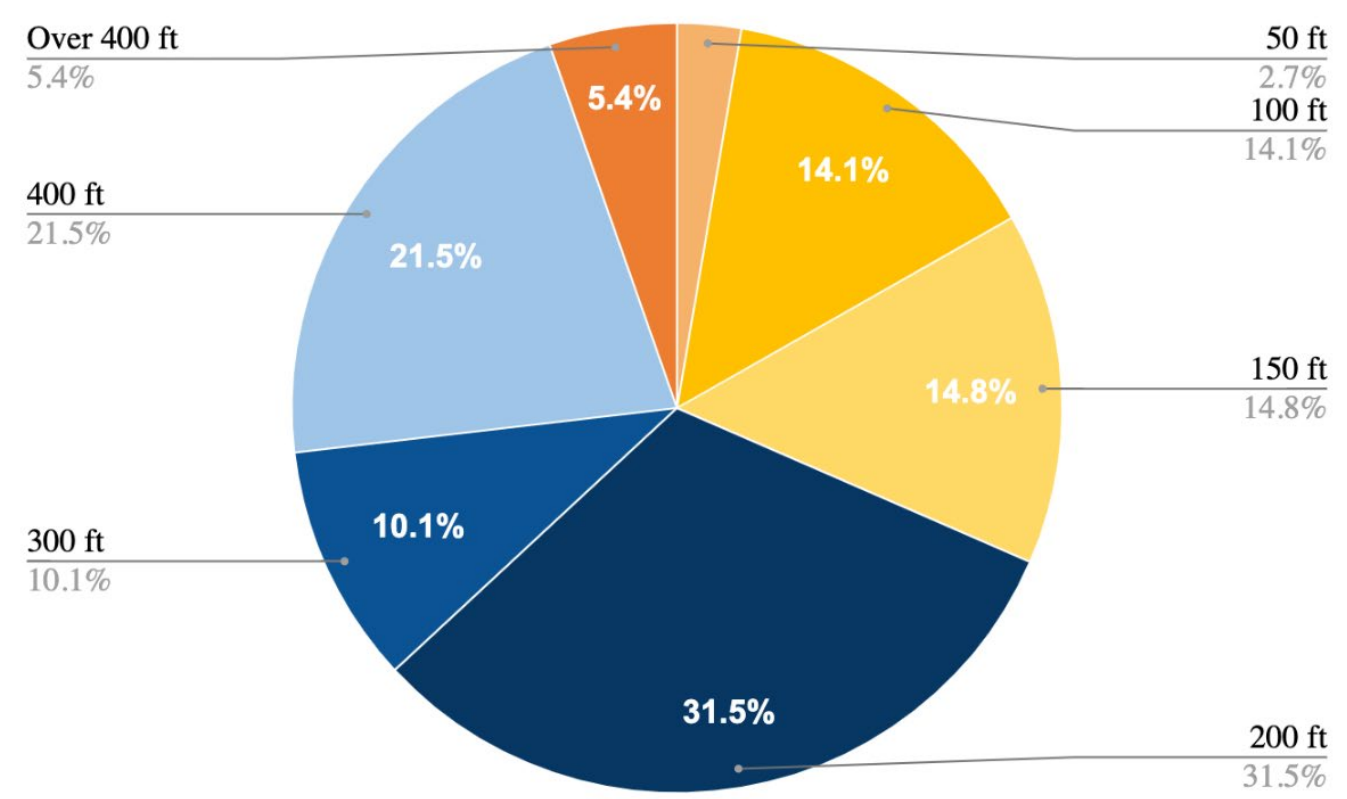

Figure 13. Displays answers to the question: "What would you consider a safe minimum flight elevation above ground level (AGL) for such a drone?" Of those surveyed, 99.5\% answered this question.

\section{Use in Various Weather Conditions}

Since first responders often conduct operations in relatively harsh or hazardous environments (e.g., heavy dew, light rain after a hurricane, dust or sand after a disaster, high humidity or smoke), respondents were asked to confirm the weather conditions that a drone might operate in when used in the described mission. The survey responses are identified in Figure 14, where the larger text indicates the most common responses about the expected weather conditions. 


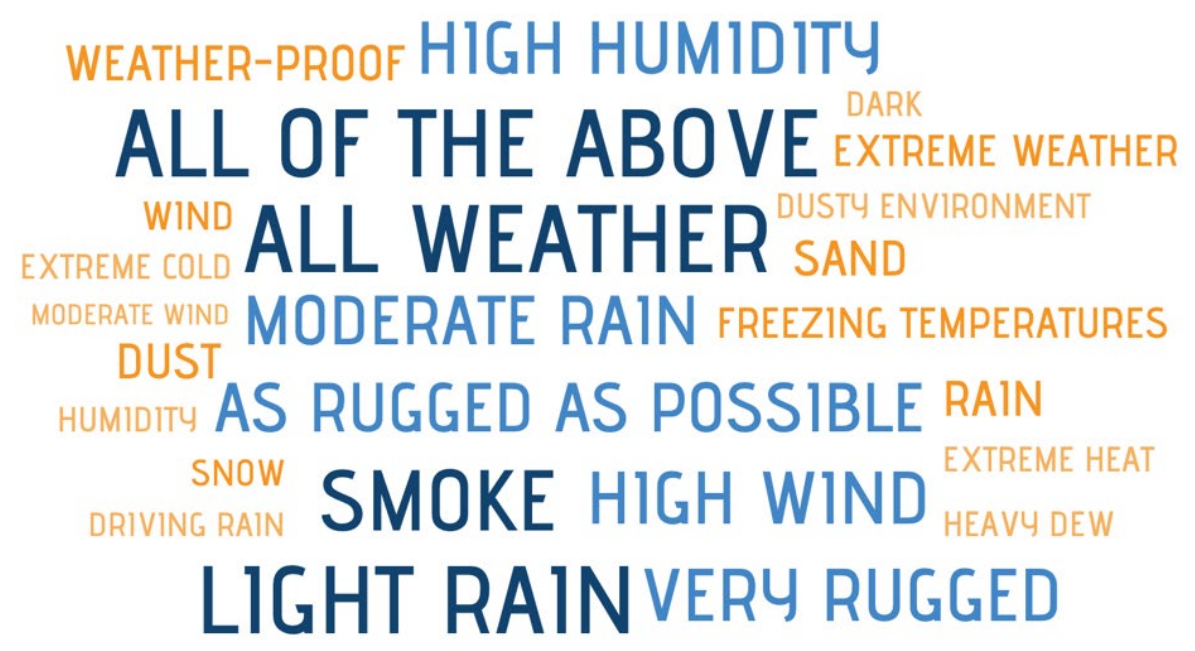

Figure 14. Respondents gave examples of weather or environmental conditions when asked the question: "For a drone that can provide continuous cellular broadband coverage for first responders, how rugged does the drone need to be? For example, would you expect to operate in weather or environmental conditions such as heavy dew, light rain after a hurricane, dust or sand after a disaster, high humidity or smoke, etc.?"

\section{Use of Cameras}

When asked about adding a high-powered zoom or thermal camera to the drone, in addition to the LTE network communications device, $89 \%$ of the respondents agreed that a camera would be useful. The respondents provided a list of camera types they might use.

- Visible and thermal

- High resolution

- LiDAR (light detection and ranging)

- Thermal imaging

- Thermal with continuous zoom

- Optical

- $\quad$ SLR (single-lens reflex)

- $\quad H D$ (high definition)

Note: $80.9 \%$ of those surveyed answered the question: "List the type of camera(s) and how it might be used (e.g., visible light camera, FLIR (thermal imaging) camera)."

Related to the use of cameras, respondents were asked if the drone would need to fly at a certain height in order to collect useful footage from the camera. Survey respondents offered a variety of explanations, indicating that 100 feet may provide useful footage for some missions, but most respondents recommended an altitude between 200 and 400 feet as the optimal height to avoid trees and other structures, and to provide better visuals of the incident area. However, many respondents indicated the height of the flying drone would be mostly dictated by the specifications of the camera and lens, and the mission's circumstances. For 
example, is the mission a surveillance, search and rescue, wildland fire or damage assessment? Any of these missions could dictate the size of the assessed area, the type of terrain as well as the type of camera or lens used. A sampling of their responses is shown below.

- As a pilot, I FULLY support a 200-foot AGL limitation in the current US airspace.

- Initial search would need $200 \mathrm{ft}$ AGL; then go lower (foliage permitting) to investigate, but no lower than $80 \mathrm{ft}$ due to $Z$ axis accuracy constraints.

- $400 \mathrm{ft}$ is the maximum needed for forest fires and sea searches.

- We regularly fly our drone missions between $200 \mathrm{ft}$ and $400 \mathrm{ft} \mathrm{AGL}$. Our flight elevation floor is adjusted based on a geofence for the area of operation.

- 400 to $500 \mathrm{ft}$ seems appropriate for factors including safety of the drones, real-time view of incidents involving apprehension of suspects, etc.

- 300 to $400 \mathrm{ft}$ is the maximum to obtain information with a zoom camera. The infrared camera needs lower altitude to get a better picture.

- Based on camera resolution, should be no more than 150-200 ft AGL.

- Live-stream video from the scene probably needs 100 to $200 \mathrm{ft}$; if SLR, maybe higher to zoom-in on a person.

- For visible light camera, anything above $100 \mathrm{ft}$ would be difficult. For FLIR with zoom and high quality, this could raise to $200 \mathrm{ft}$.

Note: $70.5 \%$ of those surveyed answered the question: "Would you need to fly at a particular maximum altitude in order to obtain useful footage from the camera(s)? Please explain." 


\section{Agency UAS Budget}

Finally, PSCR explored the potential funds that a public safety agency would spend, or consider reasonable, to purchase a drone that would assist in its mission by carrying a broadband wireless communications device. The results of the survey were scattered, showing a majority prefer spending $\$ 10,000$ or less to purchase this device or tool.

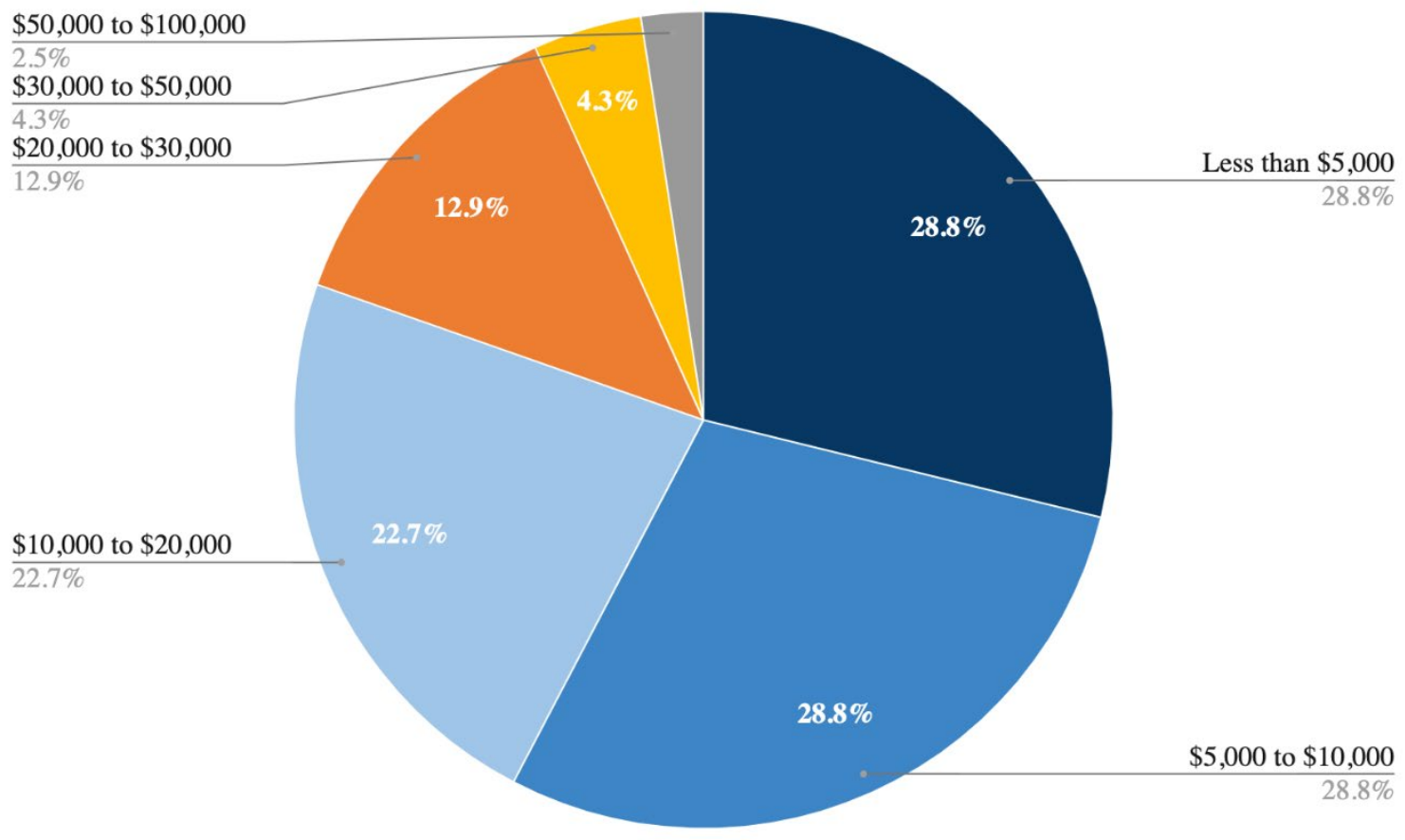

Figure 15. Displays answers to the question: "As it pertains to your agency, budget and the value to your agency of having this tool at your disposal, what would be a reasonable or affordable cost for your agency to spend on a drone to deploy cellular broadband communications?" Of those surveyed, $97.3 \%$ answered this question. 


\section{Conclusion}

This survey is an example of the collaboration between PSCR and the first responder community. In the survey, PSCR evaluated and compared answers from first responders about the availability and use of wireless communications tools today with the potential use of deployable systems in the future. The results of the survey clarified that there is still a need to maximize the flight time of a small UAS, which is categorized by the FAA to be less than 55 pounds (including payload). Additionally, it helped identify the most critical drone requirements needed by public safety when they are used to supplement or provide wireless communications infrastructure. The survey identified a potential gap in the use of drones in some public safety operations where drones might improve incident response and operational performance, but it also showed that agencies are making attempts to add UAS expertise and skilled drone pilots. The initial findings by PSCR are not intended to offer concluding statements; rather, the survey results are being made available in order to share statistics that can be used by other researchers, public safety agencies, and government entities.

The next step for PSCR is to use these survey results to inform their research in resilient systems. PSCR plans to use an alternative research method, called a prize challenge, to learn more and hopefully to improve the flight time of a UAS carrying a payload. Challenges are a unique way to find answers and solve research problems by involving external researchers and solvers to compete amongst each other for a specific timeframe. One of the many advantages of using challenges as a research method is the ability to expand the pool of problem solvers beyond traditional means, by crowdsourcing solvers from anywhere in the world. An upcoming prize challenge by PSCR is under development, with the goal of driving research in the area of UAS flight time versus payload.

NIST PSCR will continue to shape UAS research efforts and the mission of advancing wireless communications for America's first responders. The results of this survey lay an important foundation for future research and testing of resilient systems for first responders. PSCR intends to use this analysis as a reference framework and tool to inform prospective solutions in the public safety community. First responders are at the core of PSCR's mission and are the beneficiaries of PSCR's research; the feedback and insight gained from this survey are invaluable in ensuring steady progress toward solving public safety's critical needs in communications technology.

\section{Acknowledgments}

Maxwell K. Maurice (NIST/CTL), Kenneth E. Kimble (NIST/EL), Samuel L. Ray (NIST/CTL), Jennifer M. O'Brate (Corner Alliance, Inc.) 